\title{
ATTRACTIVE POINT AND MEAN CONVERGENCE THEOREMS FOR NEW GENERALIZED NONSPREADING MAPPINGS IN BANACH SPACES
}

\author{
WATARU TAKAHASHI, NGAI-CHING WONG, AND JEN-CHIH YAO
}

\begin{abstract}
In this paper, we first introduce a class of nonlinear mappings called generic generalized nonspreading which contains the class of generalized nonspreading mappings in a Banach space and then prove an attractive point theorem for such mappings in a Banach space. Furthermore, we prove a mean convergence theorem of Baillon's type and a weak convergence theorem of Mann's type for such nonlinear mappings in a Banach space. These results generalize attractive point, mean convergence and weak convergence theorems proved by Lin and Takahashi [26], and Kocourek, Takahashi and Yao [21] in a Banach space.
\end{abstract}

\section{INTRODUCTION}

Let $H$ be a real Hilbert space with inner product $\langle\cdot, \cdot\rangle$ and norm $\|\cdot\|$, respectively and let $C$ be a nonempty subset of $H$. Let $T$ be a mapping of $C$ into $H$. Then we denote by $F(T)$ the set of fixed points of $T$ and by $A(T)$ the set of attractive points [36] of $T$, i.e.,

(i) $F(T)=\{z \in C: T z=z\}$;

(ii) $A(T)=\{z \in H:\|T x-z\| \leq\|x-z\|, \forall x \in C\}$.

A mapping $T: C \rightarrow H$ is called nonexpansive if $\|T x-T y\| \leq\|x-y\|$ for all $x, y \in C$. An important example of nonexpansive mappings in a Hilbert space is a firmly nonexpansive mapping. Let $C$ be a nonempty subset of $H$. A mapping $F: C \rightarrow H$ is said to be firmly nonexpansive if

$$
\|F x-F y\|^{2} \leq\langle x-y, F x-F y\rangle
$$

for all $x, y \in C$; see, for instance, Browder [4] and Goebel and Kirk [7]. It is known that a firmly nonexpansive mapping $F$ can be deduced from an equilibrium problem in a Hilbert space; see, for instance, [3] and [5]. Recently, Kohsaka and Takahashi [24], and Takahashi [35] introduced the following nonlinear mappings which are deduced from a firmly nonexpansive mapping in a Hilbert space. A mapping $T: C \rightarrow H$ is called nonspreading [24] if

$$
2\|T x-T y\|^{2} \leq\|T x-y\|^{2}+\|T y-x\|^{2}
$$

for all $x, y \in C$. A mapping $T: C \rightarrow H$ is called hybrid [35] if

$$
3\|T x-T y\|^{2} \leq\|x-y\|^{2}+\|T x-y\|^{2}+\|T y-x\|^{2}
$$

1991 Mathematics Subject Classification. 47H10, 47H25.

Key words and phrases. Attractive point, Banach limit, Banach space, fixed point, generalized nonspreading mapping, mean convergence. 
for all $x, y \in C$. They proved fixed point theorems for such mappings; see also Kohsaka and Takahashi [23] and Iemoto and Takahashi [16]. Motivated by these mappings, Kocourek, Takahashi and Yao [20] defined a broad class of nonlinear mappings containing the classes of nonexpansive mappings, nonspreading mappings and hybrid mappings in a Hilbert space. A mapping $T: C \rightarrow H$ is called generalized hybrid [20] if there exist $\alpha, \beta \in \mathbb{R}$ such that

$$
\alpha\|T x-T y\|^{2}+(1-\alpha)\|x-T y\|^{2} \leq \beta\|T x-y\|^{2}+(1-\beta)\|x-y\|^{2}
$$

for all $x, y \in C$. We call such a mapping an $(\alpha, \beta)$-generalized hybrid mapping. Then Kocourek, Takahashi and Yao [20] proved a fixed point theorem for such mappings in a Hilbert space. Furthermore, they proved the following mean convergence theorem which generalizes Baillon's nonlinear ergodic theorem [2] in a Hilbert space.

Theorem 1.1 (Kocourek, Takahashi and Yao [20]). Let $H$ be a Hilbert space and let $C$ be a nonempty closed convex subset of $H$. Let $T: C \rightarrow C$ be a generalized hybrid mapping with $F(T) \neq \emptyset$ and let $P$ be the mertic projection of $H$ onto $F(T)$. Then for any $x \in C$,

$$
S_{n} x=\frac{1}{n} \sum_{k=0}^{n-1} T^{k} x
$$

converges weakly to an element $p \in F(T)$, where $p=\lim _{n \rightarrow \infty} P T^{n} x$.

Recently Takahashi and Takeuchi [36] proved attractive point and mean convergence theorems without convexity for generalized hybrid mappings in a Hilbert space; see also Lin and Takahashi [25]. Such theorems were also extended to Banach spaces by Kocourek, Takahashi and Yao [21] and Lin and Takahashi [26]; see also Lin, Takahashi and Yu [27]. Very recently Takahashi, Wong and Yao [37] introduced a broad class of nonlinear mappings in a Hilbert space which contains the class of contractive mappings and the class of generalized hybrid mappings. Then they proved attractive point and mean convergence theorems without convexity for such mappings in a Hilbert space. They also proved a weak convergence theorem of Mann's type [28] without closedness.

In this paper, motivated by these results, we first introduce a class of nonlinear mappings called generic generalized nonspreading which contains the class of generalized nonspreading mappings in a Banach space and then prove an attractive point theorem for such mappings in a Banach space. Furthermore, we prove a mean convergence theorem of Baillon's type and a weak convergence theorem of Mann's type for such nonlinear mappings in a Banach space. These results generalize attractive point, mean convergence and weak convergence theorems proved by Lin and Takahashi [26], and Kocourek, Takahashi and Yao [21] in a Banach space.

\section{Preliminaries}

Let $E$ be a real Banach space with norm $\|\cdot\|$ and let $E^{*}$ be the topological dual space of $E$. We denote the value of $y^{*} \in E^{*}$ at $x \in E$ by $\left\langle x, y^{*}\right\rangle$. When $\left\{x_{n}\right\}$ is a sequence in $E$, we denote the strong convergence of $\left\{x_{n}\right\}$ to $x \in E$ by $x_{n} \rightarrow x$ and the weak convergence by $x_{n} \rightarrow x$. The modulus $\delta$ of convexity of $E$ is defined by

$$
\delta(\epsilon)=\inf \left\{1-\frac{\|x+y\|}{2}:\|x\| \leq 1,\|y\| \leq 1,\|x-y\| \geq \epsilon\right\}
$$


for every $\epsilon$ with $0 \leq \epsilon \leq 2$. A Banach space $E$ is said to be uniformly convex if $\delta(\epsilon)>0$ for every $\epsilon>0$. A uniformly convex Banach space is strictly convex and reflexive. Let $C$ be a nonempty subset of a Banach space $E$. A mapping $T: C \rightarrow E$ is nonexpansive if $\|T x-T y\| \leq\|x-y\|$ for all $x, y \in C$. A mapping $T: C \rightarrow E$ is quasi-nonexpansive if $F(T) \neq \emptyset$ and $\|T x-y\| \leq\|x-y\|$ for all $x \in C$ and $y \in F(T)$, where $F(T)$ is the set of fixed points of $T$. If $C$ is a nonempty closed convex subset of a strictly convex Banach space $E$ and $T: C \rightarrow E$ is quasi-nonexpansive, then $F(T)$ is closed and convex; see Itoh and Takahashi [17]. Let $E$ be a Banach space. The duality mapping $J$ from $E$ into $2^{E^{*}}$ is defined by

$$
J x=\left\{x^{*} \in E^{*}:\left\langle x, x^{*}\right\rangle=\|x\|^{2}=\left\|x^{*}\right\|^{2}\right\}
$$

for every $x \in E$. Let $U=\{x \in E:\|x\|=1\}$. The norm of $E$ is said to be Gâteaux differentiable if for each $x, y \in U$, the limit

$$
\lim _{t \rightarrow 0} \frac{\|x+t y\|-\|x\|}{t}
$$

exists. In the case, $E$ is called smooth. We know that $E$ is smooth if and only if $J$ is a single-valued mapping of $E$ into $E^{*}$. We also know that $E$ is reflexive if and only if $J$ is surjective, and $E$ is strictly convex if and only if $J$ is one-to-one. Therefore, if $E$ is a smooth, strictly convex and reflexive Banach space, then $J$ is a single-valued bijection. The norm of $E$ is said to be uniformly Gâteaux differentiable if for each $y \in U$, the limit (2.1) is attained uniformly for $x \in U$. It is also said to be Fréchet differentiable if for each $x \in U$, the limit (2.1) is attained uniformly for $y \in U$. A Banach space $E$ is called uniformly smooth if the limit (2.1) is attained uniformly for $x, y \in U$. It is known that if the norm of $E$ is uniformly Gâteaux differentiable, then $J$ is uniformly norm-to-weak* continuous on each bounded subset of $E$, and if the norm of $E$ is Fréchet differentiable, then $J$ is norm-to-norm continuous. If $E$ is uniformly smooth, $J$ is uniformly norm-to-norm continuous on each bounded subset of $E$. For more details, see $[32,33]$. The following result is also well known; see [32].

Lemma 2.1. Let $E$ be a smooth Banach space and let $J$ be the duality mapping on $E$. Then, $\langle x-y, J x-J y\rangle \geq 0$ for all $x, y \in E$. Further, if $E$ is strictly convex and $\langle x-y, J x-J y\rangle=0$, then $x=y$.

Let $E$ be a smooth Banach space. The function $\phi: E \times E \rightarrow(-\infty, \infty)$ is defined by

$$
\phi(x, y)=\|x\|^{2}-2\langle x, J y\rangle+\|y\|^{2}
$$

for $x, y \in E$, where $J$ is the duality mapping of $E$; see [1] and [18]. We have from the definition of $\phi$ that

$$
\phi(x, y)=\phi(x, z)+\phi(z, y)+2\langle x-z, J z-J y\rangle
$$

for all $x, y, z \in E$. From $(\|x\|-\|y\|)^{2} \leq \phi(x, y)$ for all $x, y \in E$, we can see that $\phi(x, y) \geq 0$. Furthermore, we can obtain the following equality:

$$
2\langle x-y, J z-J w\rangle=\phi(x, w)+\phi(y, z)-\phi(x, z)-\phi(y, w)
$$

for $x, y, z, w \in E$. Let $\phi_{*}: E^{*} \times E^{*} \rightarrow(-\infty, \infty)$ be the function defined by

$$
\phi_{*}\left(x^{*}, y^{*}\right)=\left\|x^{*}\right\|^{2}-2\left\langle J^{-1} y^{*}, x^{*}\right\rangle+\left\|y^{*}\right\|^{2}
$$

for $x^{*}, y^{*} \in E^{*}$, where $J$ is the duality mapping of $E$. It is easy to see that

$$
\phi(x, y)=\phi_{*}(J y, J x)
$$


for $x, y \in E$. If $E$ is additionally assumed to be strictly convex, then

$$
\phi(x, y)=0 \Longleftrightarrow x=y .
$$

The following results are in Xu [41] and Kamimura and Takahashi [18].

Lemma 2.2 (Xu [41]). Let $E$ be a uniformly convex Banach space and let $r>0$. Then there exists a strictly increasing, continuous and convex function $g:[0, \infty) \rightarrow$ $[0, \infty)$ such that $g(0)=0$ and

$$
\|\lambda x+(1-\lambda) y\|^{2} \leq \lambda\|x\|^{2}+(1-\lambda)\|y\|^{2}-\lambda(1-\lambda) g(\|x-y\|)
$$

for all $x, y \in B_{r}$ and $\lambda$ with $0 \leq \lambda \leq 1$, where $B_{r}=\{z \in E:\|z\| \leq r\}$.

Lemma 2.3 (Kamimura and Takahashi [18]). Let E be smooth and uniformly convex Banach space and let $r>0$. Then there exists a strictly increasing, continuous and convex function $g:[0,2 r] \rightarrow \mathbb{R}$ such that $g(0)=0$ and

$$
g(\|x-y\|) \leq \phi(x, y)
$$

for all $x, y \in B_{r}$, where $B_{r}=\{z \in E:\|z\| \leq r\}$.

Let $E$ be a smooth Banach space. Let $C$ be a nonempty subset of $E$ and let $T$ be a mapping of $C$ into $E$. We denote by $A(T)$ the set of attractive points of $T$, i.e., $A(T)=\{z \in E: \phi(z, T x) \leq \phi(z, x), \forall x \in C\}$; see Lin and Takahashi [26]. The following result is crucial in our paper.

Lemma $2.4([26])$. Let $E$ be a smooth Banach space and let $C$ be a nonempty subset of $E$. Let $T$ be a mapping from $C$ into $E$. Then $A(T)$ is a closed and convex subset of $E$.

Let $E$ be a smooth Banach space and let $C$ be a nonempty subset of $E$. Then a mapping $T: C \rightarrow E$ is called generalized nonexpansive [12] if $F(T) \neq \emptyset$ and

$$
\phi(T x, y) \leq \phi(x, y)
$$

for all $x \in C$ and $y \in F(T)$. Let $D$ be a nonempty subset of a Banach space $E$. A mapping $R: E \rightarrow D$ is said to be sunny if

$$
R(R x+t(x-R x))=R x
$$

for all $x \in E$ and $t \geq 0$. A mapping $R: E \rightarrow D$ is said to be a retraction or a projection if $R x=x$ for all $x \in D$. A nonempty subset $D$ of a smooth Banach space $E$ is said to be a generalized nonexpansive retract (resp. sunny generalized nonexpansive retract) of $E$ if there exists a generalized nonexpansive retraction (resp. sunny generalized nonexpansive retraction) $R$ from $E$ onto $D$; see $[11,12]$ for more details. The following results are in Ibaraki and Takahashi [12].

Lemma 2.5 (Ibaraki and Takahashi [12]). Let $C$ be a nonempty closed sunny generalized nonexpansive retract of a smooth and strictly convex Banach space E. Then the sunny generalized nonexpansive retraction from $E$ onto $C$ is uniquely determined.

Lemma 2.6 (Ibaraki and Takahashi [12]). Let $C$ be a nonempty closed subset of a smooth and strictly convex Banach space $E$ such that there exists a sunny generalized nonexpansive retraction $R$ from $E$ onto $C$ and let $(x, z) \in E \times C$. Then the following hold:

(i) $z=R x$ if and only if $\langle x-z, J y-J z\rangle \leq 0$ for all $y \in C$; 
(ii) $\phi(R x, z)+\phi(x, R x) \leq \phi(x, z)$.

In 2007, Kohsaka and Takahashi [22] proved the following results:

Lemma 2.7 (Kohsaka and Takahashi [22]). Let E be a smooth, strictly convex and reflexive Banach space and let $C$ be a nonempty closed subset of $E$. Then the following are equivalent:

(a) $C$ is a sunny generalized nonexpansive retract of $E$;

(b) $C$ is a generalized nonexpansive retract of $E$;

(c) JC is closed and convex.

Lemma 2.8 (Kohsaka and Takahashi [22]). Let E be a smooth, strictly convex and reflexive Banach space and let $C$ be a nonempty closed sunny generalized nonexpansive retract of $E$. Let $R$ be the sunny generalized nonexpansive retraction from $E$ onto $C$ and let $(x, z) \in E \times C$. Then the following are equivalent:

(i) $z=R x$;

(ii) $\phi(x, z)=\min _{y \in C} \phi(x, y)$.

Recently Ibaraki and Takahashi [15] also obtained the following result concerning the set of fixed points of a generalized nonexpansive mapping.

Lemma 2.9 (Ibaraki and Takahashi [15]). Let $E$ be a smooth, strictly convex and reflexive Banach space and let $T$ be a generalized nonexpansive mapping from $E$ into itself. Then $F(T)$ is closed and $J F(T)$ is closed and convex.

The following is a direct consequence of Lemmas 2.7 and 2.9.

Lemma 2.10 (Ibaraki and Takahashi [15]). Let E be a smooth, strictly convex and reflexive Banach space and let $T$ be a generalized nonexpansive mapping from $E$ into itself. Then $F(T)$ is a sunny generalized nonexpansive retract of $E$.

Let $l^{\infty}$ be the Banach space of bounded sequences with supremum norm. Let $\mu$ be an element of $\left(l^{\infty}\right)^{*}$ (the dual space of $l^{\infty}$ ). Then we denote by $\mu(f)$ the value of $\mu$ at $f=\left(x_{1}, x_{2}, x_{3}, \ldots\right) \in l^{\infty}$. Sometimes we denote by $\mu_{n}\left(x_{n}\right)$ the value $\mu(f)$. A linear functional $\mu$ on $l^{\infty}$ is called a mean if $\mu(e)=\|\mu\|=1$, where $e=(1,1,1, \ldots)$. A mean $\mu$ is called a Banach limit on $l^{\infty}$ if $\mu_{n}\left(x_{n+1}\right)=\mu_{n}\left(x_{n}\right)$. We know that there exists a Banach limit on $l^{\infty}$. If $\mu$ is a Banach limit on $l^{\infty}$, then for $f=\left(x_{1}, x_{2}, x_{3}, \ldots\right) \in l^{\infty}$,

$$
\liminf _{n \rightarrow \infty} x_{n} \leq \mu_{n}\left(x_{n}\right) \leq \limsup _{n \rightarrow \infty} x_{n} .
$$

In particular, if $f=\left(x_{1}, x_{2}, x_{3}, \ldots\right) \in l^{\infty}$ and $x_{n} \rightarrow a \in \mathbb{R}$, then we have $\mu(f)=$ $\mu_{n}\left(x_{n}\right)=a$. See [32] for the proof of existence of a Banach limit and its other elementary properties. Using means and the Riesz theorem, we can obtain the following result; see [25], [31] and [32]. In particular, we know the following result from [31] and [8].

Lemma 2.11. Let $E$ be a reflexive Banach space, let $\left\{x_{n}\right\}$ be a bounded sequence in $E$ and let $\mu$ be a mean on $l^{\infty}$. Then there exists a unique point $z_{0} \in \overline{c o}\left\{x_{n}: n \in \mathbb{N}\right\}$ such that

$$
\mu_{n}\left\langle x_{n}, y^{*}\right\rangle=\left\langle z_{0}, y^{*}\right\rangle, \quad \forall y^{*} \in E^{*} .
$$




\section{Attractive point theorems}

In this section, we introduce a new class of nonlinear mappings called generic generalized nonspreading which contains the class of generalized nonspreading mappings in a Banach space and then try to extend Lin and Takahashi's attractive point theorem [26] for generalized nonspreading mappings to this class in a Banach space. Let $E$ be a smooth Banach space, let $C$ be a nonempty subset of $E$ and let $J$ be the duality mapping from $E$ into $E^{*}$. Then a mapping $T: C \rightarrow E$ is called widely generalized nonspreading if there exist $\alpha, \beta, \gamma, \delta, \varepsilon, \zeta \in \mathbb{R}$ such that

$$
\begin{aligned}
& \alpha \phi(T x, T y)+\beta \phi(x, T y)+\gamma \phi(T x, y)+\delta \phi(x, y) \\
& \leq \varepsilon\{\phi(T y, T x)-\phi(T y, x)\}+\zeta\{\phi(y, T x)-\phi(y, x)\}
\end{aligned}
$$

for all $x, y \in C$, where $\phi(x, y)=\|x\|^{2}-2\langle x, J y\rangle+\|y\|^{2}$ for $x, y \in E$. We call such a mapping a widely $(\alpha, \beta, \gamma, \delta, \varepsilon, \zeta)$-generalized nonspreading mapping. A widely $(\alpha, \beta, \gamma, \delta, \varepsilon, \zeta)$-generalized nonspreading mapping $T: C \rightarrow E$ is called generic generalized nonspreading if the following two conditoins are satisfied:

(1) $\alpha+\beta+\gamma+\delta \geq 0$;

(2) $\alpha+\beta>0$.

We call such a mapping a generic $(\alpha, \beta, \gamma, \delta, \varepsilon, \zeta)$-generalized nonspreading mapping. A generic $(\alpha, \beta, \gamma, \delta, \varepsilon, \zeta)$-generalized nonspreading mapping $T: C \rightarrow E$ is generalized nonspreading in the sense of Kocourek, Takahashi and Yao [21] if $\alpha+\beta=-\gamma-\delta=1$. In particular, putting $\alpha=1, \beta=\delta=0, \gamma=\varepsilon=-1$ and $\zeta=0$ in (3.1), we obtain that

$$
\phi(T x, T y)+\phi(T y, T x) \leq \phi(T x, y)+\phi(T y, x)
$$

for all $x, y \in C$. Such a mapping is nonspreading in the sense of Kohsaka and Takahashi [24]. A nonspreading mapping is deduced from a resolvent of a maximal monotone operator in a Banach space; see [24].

Now using the technique developed by [31], we prove an attractive point theorem for generic generalized nonspreading mappings in a Banach space.

Theorem 3.1. Let $E$ be a smooth and reflexive Banach space and let $C$ be a nonempty subset of $E$. Let $T$ be a generic generalized nonspreading mapping of $C$ into itself. Then the following are equivalent:

(a) $A(T) \neq \emptyset$;

(b) $\left\{T^{n} x\right\}$ is bounded for some $x \in C$.

Additionally, if $E$ is strictly convex and $C$ is closed and convex, then the following are equivalent:

(a) $F(T) \neq \emptyset$;

(b) $\left\{T^{n} x\right\}$ is bounded for some $x \in C$.

Proof. Let $T$ be a generic generalized nonspreading mapping of $C$ into itself. Then there exist $\alpha, \beta, \gamma, \delta, \varepsilon, \zeta \in \mathbb{R}$ satisfying (3.1). Furthermore, (1) $\alpha+\beta+\gamma+\delta \geq 0$ and (2) $\alpha+\beta>0$ hold. If $A(T) \neq \emptyset$, then $\phi(u, T y) \leq \phi(u, y)$ for all $u \in A(T)$ and $y \in C$. If $u$ is an attractive point, then we have that $\phi\left(u, T^{n} x\right) \leq \phi(u, x)$ for all $n \in \mathbb{N}$ and $x \in C$. This implies (a) $\Longrightarrow$ (b). Let us show (b) $\Longrightarrow$ (a). Suppose that there exists $x \in C$ such that $\left\{T^{n} x\right\}$ is bounded. Then for any $y \in C$ and 
$n \in \mathbb{N} \cup\{0\}$, we have

(3.2) $\alpha \phi\left(T^{n+1} x, T y\right)+\beta \phi\left(T^{n} x, T y\right)+\gamma \phi\left(T^{n+1} x, y\right)+\delta \phi\left(T^{n} x, y\right)$

$$
\leq \varepsilon\left\{\phi\left(T y, T^{n+1} x\right)-\phi\left(T y, T^{n} x\right)\right\}+\zeta\left\{\phi\left(y, T^{n+1} x\right)-\phi\left(y, T^{n} x\right)\right\}
$$

Since $\left\{T^{n} x\right\}$ is bounded, we can apply a Banach limit $\mu$ to both sides of the inequality. We have that

$$
(\alpha+\beta) \mu_{n} \phi\left(T^{n} x, T y\right)+(\gamma+\delta) \mu_{n} \phi\left(T^{n} x, y\right) \leq 0 .
$$

Since $\phi\left(T^{n} x, T y\right)=\phi\left(T^{n} x, y\right)+\phi(y, T y)+2\left\langle T^{n} x-y, J y-J T y\right\rangle$ from (2.2), we have that

$$
\begin{gathered}
(\alpha+\beta)\left(\mu_{n} \phi\left(T^{n} x, y\right)+\phi(y, T y)+2 \mu_{n}\left\langle T^{n} x-y, J y-J T y\right\rangle\right) \\
+(\gamma+\delta) \mu_{n} \phi\left(T^{n} x, y\right) \leq 0 .
\end{gathered}
$$

Since there exists $p \in E$ from Lemma 2.11 such that

$$
\mu_{n}\left\langle T^{n} x, v^{*}\right\rangle=\left\langle p, v^{*}\right\rangle
$$

for all $v^{*} \in E^{*}$, we have from (3.3) that

$$
\begin{aligned}
(\alpha+\beta)\left(\mu_{n} \phi\left(T^{n} x, y\right)\right. & +\phi(y, T y)+2\langle p-y, J y-J T y\rangle) \\
& +(\gamma+\delta) \mu_{n} \phi\left(T^{n} x, y\right) \leq 0
\end{aligned}
$$

and

$$
\begin{aligned}
(\alpha+\beta+\gamma+\delta) & \mu_{n} \phi\left(T^{n} x, y\right) \\
& +(\alpha+\beta)\{\phi(y, T y)+2\langle p-y, J y-J T y\rangle\} \leq 0 .
\end{aligned}
$$

From (2.3) and (1) $\alpha+\beta+\gamma+\delta \geq 0$ we obtain that

$$
(\alpha+\beta)\{\phi(y, T y)+\phi(p, T y)+\phi(y, y)-\phi(p, y)-\phi(y, T y)\} \leq 0
$$

and hence

$$
(\alpha+\beta)(\phi(p, T y)-\phi(p, y)) \leq 0 .
$$

From (2) $\alpha+\beta>0$, we have that

$$
\phi(p, T y) \leq \phi(p, y)
$$

for all $y \in C$. This implies that $p \in A(T)$.

Additionally, assume that $E$ is strictly convex and $C$ is closed and convex. Then $p \in \overline{c o}\left\{x_{n}: n \in \mathbb{N}\right\} \subset C$. Putting $y=p$ in (3.4), we obtain $\phi(p, T p)=0$. Since $E$ is strictly convex, we have $p \in F(T)$. Therefore $F(T)$ is nonempty. It is obvious that if $F(T) \neq \emptyset$, then $\left\{T^{n} x\right\}$ is bounded for every $x \in C$. This completes the proof.

Using Theorem 3.1, we have the following theorems in a Banach space.

Theorem 3.2 (Lin and Takahashi [26]). Let $E$ be a smooth and reflexive Banach space. Let $C$ be a nonempty subset of $E$ and let $T$ be a generalized nonspreading mapping of $C$ into itself, i.e., there exist $\alpha, \beta, \gamma, \delta \in \mathbb{R}$ such that

$$
\begin{aligned}
\alpha \phi(T x, T y)+(1-\alpha) \phi(x, T y)+\gamma\{\phi(T y, T x)-\phi(T y, x)\} \\
\leq \beta \phi(T x, y)+(1-\beta) \phi(x, y)+\delta\{\phi(y, T x)-\phi(y, x)\}
\end{aligned}
$$

for all $x, y \in C$. Then the following are equivalent:

(a) $A(T) \neq \emptyset$;

(b) $\left\{T^{n} x\right\}$ is bounded for some $x \in C$. 
Additionally, if $E$ is strictly convex and $C$ is closed and convex, then the following are equivalent:

(a) $F(T) \neq \emptyset$;

(b) $\left\{T^{n} x\right\}$ is bounded for some $x \in C$.

Proof. If $\alpha+\beta=-\gamma-\delta=1$ in (3.1), then the mapping is generalized nonspreading. Therefore we have the desired result from Theorem 3.1.

Theorem 3.3 (Kohsaka and Takahashi [24]). Let $E$ be a smooth, strictly convex and reflexive Banach space and let $C$ be a nonempty closed convex subset of $E$. Let $T: C \rightarrow C$ be a nonspreading mapping, i.e.,

$$
\phi(T x, T y)+\phi(T y, T x) \leq \phi(T x, y)+\phi(T y, x)
$$

for all $x, y \in C$. Then the following are equivalent:

(a) $F(T) \neq \emptyset$;

(b) $\left\{T^{n} x\right\}$ is bounded for some $x \in C$.

Proof. $\alpha=1, \beta=\delta=0, \gamma=\varepsilon=-1$ and $\zeta=0$ in (3.1), we obtain that

$$
\phi(T x, T y)+\phi(T y, T x) \leq \phi(T x, y)+\phi(T y, x)
$$

for all $x, y \in C$. Therefore we have the desired result from Theorem 3.1.

Using Theorem 3.1, we also have Takahashi, Wong and Yao's attractive point theorem [37] in a Hilbert space.

Theorem 3.4 ([37]). Let $H$ be a real Hilbert space, let $C$ be a nonempty subset of $H$ and let $T$ be a generic generalized hybrid mapping from $C$ into itself, i.e., there exist $\alpha, \beta, \gamma, \delta \in \mathbb{R}$ such that (1) $\alpha+\beta+\gamma+\delta \geq 0$, (2) $\alpha+\beta>0$ and

$$
\alpha\|T x-T y\|^{2}+\beta\|x-T y\|^{2}+\gamma\|T x-y\|^{2}+\delta\|x-y\|^{2} \leq 0
$$

for all $x, y \in C$. Then $T$ has an attractive point if and only if there exists $z \in C$ such that $\left\{T^{n} z: n=0,1, \ldots\right\}$ is bounded. Additionally, if $C$ is closed and convex, then $T$ has a fixed point if and only if there exists $z \in C$ such that $\left\{T^{n} z: n=0,1, \ldots\right\}$ is bounded.

Proof. In a Hilbert space $H$, we have that $\phi(x, y)=\|x-y\|^{2}$ for $x, y \in H$. Putting $\varepsilon=\zeta=0$ in $(3.1)$ we obtain that

$$
\alpha\|T x-T y\|^{2}+\beta\|x-T y\|^{2}+\gamma\|T x-y\|^{2}+\delta\|x-y\|^{2} \leq 0
$$

for all $x, y \in C$. Furthermore, (1) $\alpha+\beta+\gamma+\delta \geq 0$ and (2) $\alpha+\beta>0$ hold. Therefore we have the desired result from Theorem 3.1.

\section{Properties of Generic Generalized Nonspreading MapPings}

In this section, we first discuss the demiclosedness property of generic generalized nonspreading mappings in a Banach space. Let $E$ be a Banach space and let $C$ be a nonempty subset of $E$. Let $T: C \rightarrow E$ be a mapping. Then $p \in C$ is called an asymptotic fixed point of $T$ [30] if there exists $\left\{x_{n}\right\} \subset C$ such that $x_{n} \rightarrow p$ and $\lim _{n \rightarrow \infty}\left\|x_{n}-T x_{n}\right\|=0$. We denote by $\hat{F}(T)$ the set of asymptotic fixed points of $T$. A mapping $T$ of $C$ into $E$ is said to have the demiclosedness property on $C$ if $\hat{F}(T)=F(T)$. We have the following result for generic generalized nonspreading mappings in a Banach space. 
Proposition 4.1. Let E be a strictly convex Banach space with a uniformly Gâteaux differentiable norm, let $C$ be a nonempty subset of $E$ and let $T$ be a generic generalized nonspreading mapping of $C$ into $E$. If $x_{n} \rightarrow z$ and $x_{n}-T x_{n} \rightarrow 0$, then $z \in A(T)$. Additionally, if $C$ is closed and convex, then $\hat{F}(T)=F(T)$.

Proof. Since $T: C \rightarrow E$ is a generic generalized nonspreading mapping, there exist $\alpha, \beta, \gamma, \delta, \varepsilon, \zeta \in \mathbb{R}$ such that (1) $\alpha+\beta+\gamma+\delta \geq 0,(2) \alpha+\beta>0$ and

$$
\begin{aligned}
\alpha \phi(T x, T y)+\beta \phi(x, T y)+\gamma \phi(T x, y)+\delta \phi(x, y) \\
\leq \varepsilon\{\phi(T y, T x)-\phi(T y, x)\}+\zeta\{\phi(y, T x)-\phi(y, x)\}
\end{aligned}
$$

for all $x, y \in C$. Let $\left\{x_{n}\right\}$ be a sequence of $C$ such that $x_{n} \rightarrow z$ and $\lim _{n \rightarrow \infty} \| x_{n}-$ $T x_{n} \|=0$. Since the norm of $E$ is uniformly Gâteaux differentiable, the duality mapping $J$ on $E$ is uniformly norm-to-weak* continuous on each bounded subset of E; see Takahashi [33]. Thus

$$
\lim _{n \rightarrow \infty}\left\langle w, J T x_{n}-J x_{n}\right\rangle=0
$$

for all $w \in E$. On the other hand, replacing $x$ by $x_{n}$ in (4.1), we obtain that

$$
\begin{aligned}
& \alpha \phi\left(T x_{n}, T y\right)+\beta \phi\left(x_{n}, T y\right)+\gamma \phi\left(T x_{n}, y\right)+\delta \phi\left(x_{n}, y\right) \\
& \quad \leq \varepsilon\left\{\phi\left(T y, T x_{n}\right)-\phi\left(T y, x_{n}\right)\right\}+\zeta\left\{\phi\left(y, T x_{n}\right)-\phi\left(y, x_{n}\right)\right\} .
\end{aligned}
$$

Then we have from (2.2) that

$$
\begin{aligned}
& \alpha\left\{\phi\left(T x_{n}, y\right)+\phi(y, T y)+2\left\langle T x_{n}-y, J y-J T y\right\rangle\right\} \\
&+ \beta\left\{\phi\left(x_{n}, y\right)+\phi(y, T y)+2\left\langle x_{n}-y, J y-J T y\right\rangle\right\} \\
&+ \gamma \phi\left(T x_{n}, y\right)+\delta \phi\left(x_{n}, y\right) \\
& \leq \varepsilon\left\{\|T y\|^{2}-2\left\langle T y, J T x_{n}\right\rangle+\left\|T x_{n}\right\|^{2}-\|T y\|^{2}+2\left\langle T y, J x_{n}\right\rangle-\left\|x_{n}\right\|^{2}\right\} \\
&+\zeta\left\{\|y\|^{2}-2\left\langle y, J T x_{n}\right\rangle+\left\|T x_{n}\right\|^{2}-\|y\|^{2}+2\left\langle y, J x_{n}\right\rangle-\left\|x_{n}\right\|^{2}\right\} .
\end{aligned}
$$

Furthermore, we have from (4.3) that

$$
\begin{aligned}
& \alpha\left\{\phi\left(T x_{n}, y\right)-\phi\left(x_{n}, y\right)+\phi\left(x_{n}, y\right)+\phi(y, T y)+2\left\langle T x_{n}-y, J y-J T y\right\rangle\right\} \\
&+ \beta\left\{\phi\left(x_{n}, y\right)+\phi(y, T y)+2\left\langle x_{n}-y, J y-J T y\right\rangle\right\} \\
&+ \gamma\left\{\phi\left(T x_{n}, y\right)-\phi\left(x_{n}, y\right)+\phi\left(x_{n}, y\right)\right\}+\delta \phi\left(x_{n}, y\right) \\
& \leq \varepsilon\left\{\|T y\|^{2}-2\left\langle T y, J T x_{n}\right\rangle+\left\|T x_{n}\right\|^{2}-\|T y\|^{2}+2\left\langle T y, J x_{n}\right\rangle-\left\|x_{n}\right\|^{2}\right\} \\
&+\zeta\left\{\|y\|^{2}-2\left\langle y, J T x_{n}\right\rangle+\left\|T x_{n}\right\|^{2}-\|y\|^{2}+2\left\langle y, J x_{n}\right\rangle-\left\|x_{n}\right\|^{2}\right\} .
\end{aligned}
$$

From (1) $\alpha+\beta+\gamma+\delta \geq 0$ we have that

$$
\begin{aligned}
& \alpha\left\{\phi\left(T x_{n}, y\right)-\phi\left(x_{n}, y\right)+\phi(y, T y)+2\left\langle T x_{n}-y, J y-J T y\right\rangle\right\} \\
&+\left.\beta\left\{\phi(y, T y)+2\left\langle x_{n}-y, J y-J T y\right\rangle\right\}+\gamma\left\{\phi\left(T x_{n}, y\right)-\phi\left(x_{n}, y\right)\right\}\right\} \\
& \leq \varepsilon\left\{\|T y\|^{2}-2\left\langle T y, J T x_{n}\right\rangle+\left\|T x_{n}\right\|^{2}-\|T y\|^{2}+2\left\langle T y, J x_{n}\right\rangle-\left\|x_{n}\right\|^{2}\right\} \\
&+\zeta\left\{\|y\|^{2}-2\left\langle y, J T x_{n}\right\rangle+\left\|T x_{n}\right\|^{2}-\|y\|^{2}+2\left\langle y, J x_{n}\right\rangle-\left\|x_{n}\right\|^{2}\right\} .
\end{aligned}
$$

Since

$$
\begin{aligned}
\left|\left\|T x_{n}\right\|^{2}-\left\|x_{n}\right\|^{2}\right| & =\left(\left\|T x_{n}\right\|+\left\|x_{n}\right\|\right)\left|\left\|T x_{n}\right\|-\left\|x_{n}\right\|\right| \\
& \leq\left(\left\|T x_{n}\right\|+\left\|x_{n}\right\|\right)\left\|T x_{n}-x_{n}\right\|
\end{aligned}
$$


and $T x_{n}-x_{n} \rightarrow 0$, we have that $\left\|T x_{n}\right\|^{2}-\left\|x_{n}\right\|^{2} \rightarrow 0$. Letting $n \rightarrow \infty$ in (4.5), we have that

$$
\begin{aligned}
& \alpha\{\phi(y, T y)+2\langle z-y, J y-J T y\rangle\} \\
& \quad+\beta\{\phi(y, T y)+2\langle z-y, J y-J T y\rangle\} \leq 0
\end{aligned}
$$

and hence

$$
(\alpha+\beta) \phi(y, T y)+2(\alpha+\beta)\langle z-y, J y-J T y\rangle \leq 0 .
$$

Thus we have from (2.3) that

$$
(\alpha+\beta) \phi(y, T y)+(\alpha+\beta)(\phi(z, T y)-\phi(z, y)-\phi(y, T y)) \leq 0
$$

and hence

$$
(\alpha+\beta)(\phi(z, T y)-\phi(z, y)) \leq 0 .
$$

From (2) $\alpha+\beta>0$, we have $z \in A(T)$. Additionally, if $C$ is closed and convex, then $z \in C$. Thus we have $\phi(z, T z) \leq 0$ and hence $\phi(z, T z)=0$. Since $E$ is strictly convex, we obtain $z=T z$. Therefore we have $\hat{F}(T) \subset F(T)$. The inclusion $F(T) \subset \hat{F}(T)$ is obvious. This completes the proof.

From Matsushita and Takahashi [29], we know the following result.

Lemma 4.2 (Matsushita and Takahashi [29]). Let $E$ be a smooth and strictly convex Banach space, let $C$ be a nonempty closed convex subset of $E$ and let $T$ be a mapping of $C$ into $E$ such that $F(T)$ is nonempty. Assume that

$$
\phi(u, T y) \leq \phi(u, y)
$$

for all $u \in F(T)$ and $y \in C$. Then $F(T)$ is closed and convex.

Using Matsushita and Takahashi [29], we have the following result.

Proposition 4.3. Let $E$ be a smooth and strictly convex Banach space, let $C$ be a nonempty closed convex subset of $E$ and let $T$ be a generic generalized nonspreading mapping of $C$ into $E$ such that $F(T)$ is nonempty. Then $F(T)$ is closed and convex.

Proof. Let $T: C \rightarrow E$ be a generic generalized nonspreading mapping satisfying (3.1). Putting $x=u \in F(T)$ in (3.1), we have that

$$
\begin{aligned}
& \alpha \phi(u, T y)+\beta \phi(u, T y)+\gamma \phi(u, y)+\delta \phi(u, y) \\
& \leq \varepsilon\{\phi(T y, u)-\phi(T y, u)\}+\zeta\{\phi(y, u)-\phi(y, u)\}
\end{aligned}
$$

and hence

$$
(\alpha+\beta) \phi(u, T y) \leq-(\gamma+\delta) \phi(u, y) .
$$

Since (1) $\alpha+\beta+\gamma+\delta \geq 0$ and $\alpha+\beta>0$, we have

$$
\phi(u, T y) \leq \frac{-(\gamma+\delta)}{\alpha+\beta} \phi(u, y) \leq \phi(u, y) .
$$

Using Matsushita and Takahashi (Lemma 4.2), we have the desired result.

Let $E$ be a smooth and strictly convex Banach space. Let $C$ be a nonempty subset of $E$. Matsushita and Takahashi [29] also gave the following definition: A mapping $T: C \rightarrow E$ is relatively nonexpansive if $F(T) \neq \emptyset, \hat{F}(T)=F(T)$ and

$$
\phi(y, T x) \leq \phi(y, x)
$$

for all $x \in C$ and $y \in F(T)$. Using Proposition 4.1, we prove the following theorem. 
Theorem 4.4. Let $E$ be a strictly convex Banach space with a uniformly Gâteaux differentiable norm, let $C$ be a nonempty closed convex subset of $E$ and let $T$ be a generic generalized nonspreading mapping of $C$ into $E$ such that $F(T)$ is nonempty. Then $T$ is relatively nonexpansive.

Proof. By assumption, $F(T)$ is nonempty. Since $T$ is a generic generalized nonspreading mapping of $C$ into $E$, we have that

$$
\phi(y, T x) \leq \phi(y, x)
$$

for all $x \in C$ and $y \in F(T)$. From Proposition 4.1, we also have $\hat{F}(T)=F(T)$. Thus $T$ is relatively nonexpansive.

As a direct consequence of Theorem 4.4, we have the following result.

Theorem 4.5 (Kohsaka and Takahashi [24]). Let E be a strictly convex Banach space with a uniformly Gâteaux differentiable norm, let $C$ be a nonempty closed convex subset of $E$ and let $T$ be a nonspreading mapping of $C$ into $E$ such that $F(T)$ is nonempty. Then $T$ is relatively nonexpansive.

Proof. A generic $(\alpha, \beta, \gamma, \delta, \varepsilon, \zeta)$-generalized hybrid mapping $T$ of $C$ into $E$ such that $\alpha=1, \beta=\delta=0, \gamma=\varepsilon=-1$ and $\zeta=0$ is a nonspreading mapping. From Theorem 4.4, we have the desired result.

\section{Skew-Attractive Point Theorems}

Let $E$ be a smooth Banach space and let $C$ be a nonempty subset of $E$. Let $T: C \rightarrow E$ be a generic generalized nonspreading mapping; see (3.1). This mapping has the property that $\phi(u, T y) \leq \phi(u, y)$ for all $u \in F(T)$ and $y \in C$. This property can be revealed by putting $x=u \in F(T)$ in (3.1); see the proof of Proposition 4.3. Similarly, putting $y=u \in F(T)$ in (3.1), we obtain that for any $x \in C$,

$$
\begin{aligned}
\alpha \phi(T x, u) & +\beta \phi(x, u)+\gamma \phi(T x, u)+\delta \phi(x, u) \\
\leq & \varepsilon\{\phi(u, T x)-\phi(u, x)\}+\zeta\{\phi(u, T x)-\phi(u, x)\}
\end{aligned}
$$

and hence

$$
\begin{aligned}
\alpha\{\phi(T x, u) & -\phi(x, u)+\phi(x, u)\}+\beta \phi(x, u) \\
+ & \gamma\{\phi(T x, u)-\phi(x, u)+\phi(x, u)\}+\delta \phi(x, u) \\
& \leq(\varepsilon+\zeta)\{\phi(u, T x)-\phi(u, x)\} .
\end{aligned}
$$

From (1) $\alpha+\beta+\gamma+\delta \geq 0$, we have that

$$
\begin{gathered}
\alpha\{\phi(T x, u)-\phi(x, u)\}+\gamma\{\phi(T x, u)-\phi(x, u)\} \\
\leq(\varepsilon+\zeta)\{\phi(u, T x)-\phi(u, x)\}
\end{gathered}
$$

and hence

$$
(\alpha+\gamma)\{\phi(T x, u)-\phi(x, u)\} \leq(\varepsilon+\zeta)\{\phi(u, T x)-\phi(u, x)\} .
$$

Since $\phi(u, T x) \leq \phi(u, x)$, we have that $\alpha+\gamma>0$ together with $\varepsilon+\zeta \geq 0$ implies that

$$
\phi(T x, u) \leq \phi(x, u) .
$$

Motivated by this property of $T$ and $F(T)$, we can give the following definition. Let $E$ be a smooth Banach space. Let $C$ be a nonempty subset of $E$ and let $T$ be a mapping of $C$ into $E$. We denote by $B(T)$ the set of skew-attractive points of 
$T$, i.e., $B(T)=\{z \in E: \phi(T x, z) \leq \phi(x, z), \forall x \in C\}$; see Lin and Takahashi [26]. They [26] proved the following result.

Lemma 5.1 ([26]). Let $E$ be a smooth Banach space and let $C$ be a nonempty subset of $E$. Let $T$ be a mapping from $C$ into $E$. Then $B(T)$ is closed.

Let $E$ be a smooth, strictly convex and reflexive Banach space and let $C$ be a nonempty subset of $E$. Let $T$ be a mapping of $C$ into $E$. Define a mapping $T^{*}$ as follows:

$$
T^{*} x^{*}=J T J^{-1} x^{*}, \quad \forall x^{*} \in J C,
$$

where $J$ is the duality mapping on $E$ and $J^{-1}$ is the duality mapping on $E^{*}$. A mapping $T^{*}$ is called the duality mapping of $T$; see also [39] and [9]. It is easy to show that if $T$ is a mapping of $C$ into itself, then $T^{*}$ is a mapping of $J C$ into itself. In fact, for any $x^{*} \in J C$, we have $J^{-1} x^{*} \in C$ and hence $T J^{-1} x^{*} \in C$ from the property of $T$. So we have

$$
T^{*} x^{*}=J T J^{-1} x^{*} \in J C .
$$

Then $T^{*}$ is a mapping of $J C$ into itself. Lin and Takahashi [26] also proved the following result by using the duality mapping $T^{*}$ of $T$ in a Banach space.

Lemma $5.2([26])$. Let $E$ be a smooth, strictly convex and reflexive Banach space and let $C$ be a nonempty subset of $E$. Let $T$ be a mapping of $C$ into $E$ and let $T^{*}$ be the duality mapping of $T$. Then the following hold:

(1) $J B(T)=A\left(T^{*}\right)$;

(2) $J A(T)=B\left(T^{*}\right)$.

In particular, $J B(T)$ is closed and convex.

Let $E$ be a smooth Banach space, let $J$ be the duality mapping from $E$ into $E^{*}$ and let $C$ be a nonempty subset of $E$. A mapping $T: C \rightarrow E$ is called widely skew-generalized nonspreading if there exist $\alpha, \beta, \gamma, \delta, \varepsilon, \zeta \in \mathbb{R}$ such that

$$
\begin{aligned}
& \alpha \phi(T y, T x)+\beta \phi(T y, x)+\gamma \phi(y, T x)+\delta \phi(y, x) \\
& \quad \leq \varepsilon\{\phi(T x, T y)-\phi(x, T y)\}+\zeta\{\phi(T x, y)-\phi(x, y)\}
\end{aligned}
$$

for all $x, y \in C$, where $\phi(x, y)=\|x\|^{2}-2\langle x, J y\rangle+\|y\|^{2}$ for $x, y \in E$. We call such a mapping a widely $(\alpha, \beta, \gamma, \delta, \varepsilon, \zeta)$-skew-generalized nonspreading mapping. A widely $(\alpha, \beta, \gamma, \delta, \varepsilon, \zeta)$-skew-generalized nonspreading mapping $T: C \rightarrow E$ is called generic skew-generalized nonspreading if the following two conditoins are satisfied:

(1) $\alpha+\beta+\gamma+\delta \geq 0$

(2) $\alpha+\beta>0$.

We call such a mapping a generic $(\alpha, \beta, \gamma, \delta, \varepsilon, \zeta)$-skew-generalized nonspreading mapping. For example, a generic $(1,0,-1,0,-1,0)$-skew-generalized nonspreading mapping is a skew-nonspreading mapping in the sense of Ibaraki and Takahashi [14], i.e.,

$$
\phi(T x, T y)+\phi(T y, T x) \leq \phi(x, T y)+\phi(y, T x), \quad \forall x, y \in C .
$$

Theorem 5.3. Let $E$ be a smooth, strictly convex and reflexive Banach space and let $C$ be a nonempty subset of $E$. Let $T$ be a generic skew-generalized nonspreading mapping of $C$ into itself. Then the following are equivalent:

(a) $B(T) \neq \emptyset$; 
(b) $\left\{T^{n} x\right\}$ is bounded for some $x \in C$.

Additionally, if $C$ is closed and $J C$ is closed and convex, then the following are equivalent:

(a) $F(T) \neq \emptyset$;

(b) $\left\{T^{n} x\right\}$ is bounded for some $x \in C$.

Proof. Let $T$ be a generic skew-generalized nonspreading mapping of $C$ into itself. Then there exist $\alpha, \beta, \gamma, \delta, \varepsilon, \zeta \in \mathbb{R}$ such that (1) $\alpha+\beta+\gamma+\delta \geq 0,(2) \alpha+\beta>0$ and

$$
\begin{aligned}
& \alpha \phi(T y, T x)+\beta \phi(T y, x)+\gamma \phi(y, T x)+\delta \phi(y, x) \\
& \leq \varepsilon\{\phi(T x, T y)-\phi(x, T y)\}+\zeta\{\phi(T x, y)-\phi(x, y)\}
\end{aligned}
$$

for all $x, y \in C$. If $B(T) \neq \emptyset$, then $\phi(T y, u) \leq \phi(y, u)$ for all $u \in B(T)$ and $y \in C$. If $u \in B(T)$, then we have that $\phi\left(T^{n} x, u\right) \leq \phi(x, u)$ for all $n \in \mathbb{N}$ and $x \in C$. This implies (a) $\Longrightarrow(\mathrm{b})$. Let us show (b) $\Longrightarrow$ (a). Suppose that there exists $x \in C$ such that $\left\{T^{n} x\right\}$ is bounded. Then for any $x^{*}, y^{*} \in J C$ with $x^{*}=J x$ and $y^{*}=J y$ and $T^{*}=J T J^{-1}$, we have from (2.4) that

$$
\begin{aligned}
& \alpha \phi_{*}\left(T^{*} x^{*}, T^{*} y^{*}\right)+\beta \phi_{*}\left(x^{*}, T^{*} y^{*}\right)+\gamma \phi_{*}\left(T^{*} x^{*}, y^{*}\right)+\delta \phi_{*}\left(x^{*}, y^{*}\right) \\
& \quad=\alpha \phi_{*}(J T x, J T y)+\beta \phi_{*}(J x, J T y)+\gamma \phi_{*}(J T x, J y)+\delta \phi_{*}(J x, J y) \\
& \quad=\alpha \phi(T y, T x)+\beta \phi(T y, x)+\gamma \phi(y, T x)+\delta \phi(y, x) .
\end{aligned}
$$

On the other hand, we have

$$
\begin{aligned}
& \varepsilon\left\{\phi_{*}\left(T^{*} y^{*}, T^{*} x^{*}\right)-\phi_{*}\left(T^{*} y^{*}, x^{*}\right)\right\}+\zeta\left\{\phi_{*}\left(y^{*}, T^{*} x^{*}\right)-\phi_{*}\left(y^{*}, x^{*}\right)\right\} \\
& \quad=\varepsilon\left\{\phi_{*}(J T y, J T x)-\phi_{*}(J T y, J x)\right\}+\zeta\left\{\phi_{*}(J y, J T x)-\phi_{*}(J y, J x)\right\} \\
& \quad=\varepsilon\{\phi(T x, T y)-\phi(x, T y)\}+\zeta\{\phi(T x, y)-\phi(x, y)\} .
\end{aligned}
$$

Since $T$ is generic skew-generalized nonspreading, we have from (5.3) that

$$
\begin{aligned}
& \alpha \phi_{*}\left(T^{*} x^{*}, T^{*} y^{*}\right)+\beta \phi_{*}\left(x^{*}, T^{*} y^{*}\right)+\gamma \phi_{*}\left(T^{*} x^{*}, y^{*}\right)+\delta \phi_{*}\left(x^{*}, y^{*}\right) \\
& \quad \leq \varepsilon\left\{\phi_{*}\left(T^{*} y^{*}, T^{*} x^{*}\right)-\phi_{*}\left(T^{*} y^{*}, x^{*}\right)\right\}+\zeta\left\{\phi_{*}\left(y^{*}, T^{*} x^{*}\right)-\phi_{*}\left(y^{*}, x^{*}\right)\right\} .
\end{aligned}
$$

This implies that $T^{*}$ is a generic generalized nonspreading mapping of $J C$ into itself. Furthermore, we have that

$$
\left(J T J^{-1}\right)^{n} J x=J T^{n} x
$$

for each $x \in C$ and $n \in \mathbb{N}$; see the proof of Lemma 3.1 in [6]. Thus if $\left\{T^{n} x\right\}$ is bounded for some $x \in C$, then $\left\{\left(T^{*}\right)^{n} J x\right\}$ is bounded. We have from Theorem 3.1 that $A\left(T^{*}\right)$ is nonempty. We also know from Lemma 5.2 that $A\left(T^{*}\right)=J B(T)$. Therefore $B(T)$ is nonempty. Additionally, assume that $C$ is closed and $J C$ is closed and convex. If $\left\{T^{n} x\right\}$ is bounded for some $x \in C$, then $\left\{\left(T^{*}\right)^{n} J x\right\}$ is bounded. Then we have from Theorem 3.1 that $F\left(T^{*}\right)$ is nonempty. We also have that $J F(T)=F\left(T^{*}\right)$. In fact, we have that for $z^{*} \in J C$,

$$
\begin{aligned}
& z^{*} \in J F(T) \Longleftrightarrow J^{-1} z^{*} \in F(T) \Longleftrightarrow T J^{-1} z^{*}=J^{-1} z^{*} \\
& \Longleftrightarrow J T J^{-1} z^{*}=z^{*} \Longleftrightarrow T^{*} z^{*}=z^{*} \Longleftrightarrow z^{*} \in F\left(T^{*}\right) .
\end{aligned}
$$

Therefore $F(T)$ is nonempty. The converse is obvious. This completes the proof.

Using Theorem 5.3, we have the following skew-attractive point theorem in a Banach space obtained by Lin and Takahashi [26]. 
Theorem $5.4([26])$. Let $E$ be a smooth, strictly convex and reflexive Banach space and let $C$ be a nonempty subset of $E$. Let $T$ be a skew-generalized nonspreading mapping of $C$ into itself. Then the following are equivalent:

(a) $B(T) \neq \emptyset$;

(b) $\left\{T^{n} x\right\}$ is bounded for some $x \in C$.

Additionally, if $C$ is closed and $J C$ is closed and convex, then the following are equivalent:

(a) $F(T) \neq \emptyset$;

(b) $\left\{T^{n} x\right\}$ is bounded for some $x \in C$.

Proof. If $\alpha+\beta=-\gamma-\delta=1$ in (5.2), then the mapping is skew-generalized nonspreading. Therefore we have the desired result from Theorem 5.3.

Using Theorem 5.3, we have the following skew-attractive point theorems in a Banach space which is a generalization of Dhompongsa, Fupinwong, Takahashi and Yao [6].

Theorem 5.5. Let $E$ be a smooth, strictly convex and reflexive Banach space and let $C$ be a nonempty subset of $E$. Let $T: C \rightarrow C$ be a skew-nonspreading mapping, i.e.,

$$
\phi(T y, T x)+\phi(T x, T y) \leq \phi(y, T x)+\phi(x, T y)
$$

for all $x, y \in C$. Then the following are equivalent:

(a) $B(T) \neq \emptyset$;

(b) $\left\{T^{n} x\right\}$ is bounded for some $x \in C$.

Proof. Putting $\alpha=1, \beta=\delta=\zeta=0$ and $\gamma=\varepsilon=-1$ in (5.2), we obtain that

$$
\phi(T y, T x)+\phi(T x, T y) \leq \phi(y, T x)+\phi(x, T y)
$$

for all $x, y \in C$. Therefore we have the desired result from Theorem 5.3.

\section{NONLINEAR ERGODIC THEOREMS}

In this section, we prove a mean convergence theorem of Baillon's type for generic generalized nonspreading mappings in a Banach space. Before proving this result, we need the following lemma.

Lemma 6.1. Let $E$ be a smooth and uniformly convex Banach space and let $C$ be a nonempty subset of $E$. Let $T: C \rightarrow C$ be a mapping such that $B(T) \neq \emptyset$. Let $R$ be the sunny generalized nonexpansive retraction of $E$ onto $B(T)$. Then for any $x \in C,\left\{R T^{n} x\right\}$ converges strongly to an element $q \in B(T)$

Proof. We know from Lemmas 5.1 and 5.2 that $B(T)$ is closed, and $J B(T)$ is closed and convex. Thus from Lemma 2.7 there exists the sunny generalized nonexpansive retraction $R$ of $E$ onto $B(T)$. From Lemma 2.8, this retraction $R$ is characterized by

$$
R x=\arg \min _{u \in B(T)} \phi(x, u) .
$$

We also know from Lemma 2.6 that for all $u \in B(T)$ and $v \in C$,

$$
0 \leq\langle v-R v, J R v-J u\rangle
$$

and

$$
\phi(R v, u)=\phi(v, u)-\phi(v, R v) .
$$


Since $\phi(T z, u) \leq \phi(z, u)$ for any $u \in B(T)$ and $z \in C$, it follows that

$$
\begin{aligned}
\phi\left(T^{n} x, R T^{n} x\right) & \leq \phi\left(T^{n} x, R T^{n-1} x\right) \\
& \leq \phi\left(T^{n-1} x, R T^{n-1} x\right) .
\end{aligned}
$$

Hence the sequence $\phi\left(T^{n} x, R T^{n} x\right)$ is nonincreasing. Putting $u=R T^{n} x$ and $v=$ $T^{m} x$ with $n \leq m$ in (6.1), we have from Lemma 2.3 that

$$
\begin{aligned}
g\left(\left\|R T^{m} x-R T^{n} x\right\|\right) & \leq \phi\left(R T^{m} x, R T^{n} x\right) \\
& \leq \phi\left(T^{m} x, R T^{n} x\right)-\phi\left(T^{m} x, R T^{m} x\right) \\
& \leq \phi\left(T^{n} x, R T^{n} x\right)-\phi\left(T^{m} x, R T^{m} x\right),
\end{aligned}
$$

where $g$ is a strictly increasing, continuous and convex real-valued function with $g(0)=0$. From the properties of $g,\left\{R T^{n} x\right\}$ is a Cauchy sequence. Therefore $\left\{R T^{n} x\right\}$ converges strongly to a point $q \in B(T)$ since $B(T)$ is closed from Lemma 5.1 .

Using Lemma 6.1, we can prove the following result for generic generalized nonspreading mappings in a Banach space.

Theorem 6.2. Let $E$ be a uniformly convex Banach space with a Fréchet differentiable norm and let $C$ be a nonempty subset of $E$. Let $T: C \rightarrow C$ be a generic generalized nonspreading mapping such that $A(T)=B(T) \neq \emptyset$. Let $R$ be the sunny generalized nonexpansive retraction of $E$ onto $B(T)$. Then for any $x \in C$,

$$
S_{n} x=\frac{1}{n} \sum_{k=0}^{n-1} T^{k} x
$$

converges weakly to an element $q \in A(T)$, where $q=\lim _{n \rightarrow \infty} R T^{n} x$. Additionally, if $C$ is closed and convex, then $\left\{S_{n} x\right\}$ converges weakly to an element $q \in F(T)$.

Proof. Take a fixed $x \in C$. Then for any $y \in C$ and $k \in \mathbb{N} \cup\{0\}$, we have that

$$
\begin{aligned}
\alpha \phi\left(T^{k+1} x, T y\right)+\beta \phi\left(T^{k} x, T y\right)+\gamma \phi\left(T^{k+1} x, y\right)+\delta \phi\left(T^{k} x, y\right) \\
\leq \varepsilon\left\{\phi\left(T y, T^{k+1} x\right)-\phi\left(T y, T^{k} x\right)\right\}+\zeta\left\{\phi\left(y, T^{k+1} x\right)-\phi\left(y, T^{k} x\right)\right\} .
\end{aligned}
$$

Since

$$
\begin{aligned}
\beta \phi\left(T^{k} x, T y\right)= & (\alpha+\beta) \phi\left(T^{k} x, T y\right)-\alpha \phi\left(T^{k} x, T y\right) \\
= & (\alpha+\beta)\left\{\phi\left(T^{k} x, y\right)+\phi(y, T y)\right. \\
& \left.+2\left\langle T^{k} x-y, J y-J T y\right\rangle\right\}-\alpha \phi\left(T^{k} x, T y\right) \\
\geq & (-\gamma-\delta) \phi\left(T^{k} x, y\right)+(\alpha+\beta)\{\phi(y, T y) \\
& \left.+2\left\langle T^{k} x-y, J y-J T y\right\rangle\right\}-\alpha \phi\left(T^{k} x, T y\right),
\end{aligned}
$$

we have that

$$
\begin{aligned}
\alpha\left\{\phi\left(T^{k+1} x, T y\right)\right. & \left.-\phi\left(T^{k} x, T y\right)\right\}+\gamma\left\{\phi\left(T^{k+1} x, y\right)-\phi\left(T^{k} x, y\right)\right\} \\
& +(\alpha+\beta)\left\{\phi(y, T y)+2\left\langle T^{k} x-y, J y-J T y\right\rangle\right\} \\
\leq & \varepsilon\left\{\phi\left(T y, T^{k+1} x\right)-\phi\left(T y, T^{k} x\right)\right\}+\zeta\left\{\phi\left(y, T^{k+1} x\right)-\phi\left(y, T^{k} x\right)\right\} .
\end{aligned}
$$


Summing up these inequalities with respect to $k=0,1, \ldots, n-1$ and dividing by $n$, we obtain that

$$
\begin{aligned}
\frac{1}{n} \alpha\left\{\phi\left(T^{n} x, T y\right)\right. & -\phi(x, T y)\}+\frac{1}{n} \gamma\left\{\phi\left(T^{n} x, y\right)-\phi(x, y)\right\} \\
+ & (\alpha+\beta)\left\{\phi(y, T y)+2\left\langle S_{n} x-y, J y-J T y\right\rangle\right\} \\
\leq & \frac{1}{n} \varepsilon\left\{\phi\left(T y, T^{n} x\right)-\phi(T y, x)\right\}+\frac{1}{n} \zeta\left\{\phi\left(y, T^{n} x\right)-\phi(y, x)\right\}
\end{aligned}
$$

where $S_{n} x=\frac{1}{n} \sum_{k=0}^{n-1} T^{k} x$. Since $\left\{T^{n} x\right\}$ is bounded, $\left\{S_{n} x\right\}$ is bounded. Thus we have a subsequence $\left\{S_{n_{i}} x\right\}$ of $\left\{S_{n} x\right\}$ such that $\left\{S_{n_{i}} x\right\}$ converges weakly to a point $u \in E$. Replacing $n$ by $n_{i}$, we have that

$$
\begin{aligned}
\frac{1}{n_{i}} \alpha\left\{\phi\left(T^{n_{i}} x, T y\right)\right. & -\phi(x, T y)\}+\frac{1}{n_{i}} \gamma\left\{\phi\left(T^{n_{i}} x, y\right)-\phi(x, y)\right\} \\
+ & (\alpha+\beta)\left\{\phi(y, T y)+2\left\langle S_{n_{i}} x-y, J y-J T y\right\rangle\right\} \\
\leq & \frac{1}{n_{i}} \varepsilon\left\{\phi\left(T y, T^{n_{i}} x\right)-\phi(T y, x)\right\}+\frac{1}{n_{i}} \zeta\left\{\phi\left(y, T^{n_{i}} x\right)-\phi(y, x)\right\} .
\end{aligned}
$$

Letting $i \rightarrow \infty$, we obtain that

$$
(\alpha+\beta)\{\phi(y, T y)+2\langle u-y, J y-J T y\rangle\} \leq 0 .
$$

Using (2.3), we obtain that

$$
(\alpha+\beta)\{\phi(y, T y)+\phi(u, T y)-\phi(u, y)-\phi(y, T y)\} \leq 0 .
$$

Hence we have $\phi(u, T y) \leq \phi(u, y)$ and then $u \in A(T)$. Rewriting the characterization of the retraction $R$, we have that for any $v \in B(T)$,

$$
0 \leq\left\langle T^{k} x-R T^{k} x, J R T^{k} x-J v\right\rangle .
$$

So putting $q=\lim _{n \rightarrow \infty} R T^{n} x$, we have that

$$
\begin{aligned}
\left\langle T^{k} x-R T^{k} x,\right. & J v-J q\rangle \leq\left\langle T^{k} x-R T^{k} x, J R T^{k} x-J q\right\rangle \\
& \leq\left\|T^{k} x-R T^{k} x\right\| \cdot\left\|J R T^{k} x-J q\right\| \\
& \leq K\left\|J R T^{k} x-J q\right\|
\end{aligned}
$$

where $K$ is an upper bound for $\left\|T^{k} x-R T^{k} x\right\|$. Summing up these inequalities for $k=0,1, \ldots, n-1$, we arrive to

$$
\left\langle S_{n} x-\frac{1}{n} \sum_{k=0}^{n-1} R T^{k} x, J v-J q\right\rangle \leq K \frac{1}{n} \sum_{k=0}^{n-1}\left\|J R T^{k} x-J q\right\| .
$$

Suppose that $\left\{S_{n_{i}} x\right\}$ converges weakly to a point $u$. Remembering that $J$ is continuous, we get

$$
\langle u-q, J v-J q\rangle \leq 0, \forall v \in B(T)
$$

Putting $v=u$ because $u \in A(T)=B(T)$, we have $u=q$. Thus the sequence $\left\{S_{n} x\right\}$ converges weakly to the point $q$. Additionally, if $C$ is closed and convex, then $\left\{S_{n} x\right\} \subset C$ and hence $q \in C$. Since $q \in A(T)$ and $q \in C$ we have $q \in F(T)$. Therefore $\left\{S_{n} x\right\}$ converges weakly to an element $q \in F(T)$.

Using Theorem 6.2, we obtain the following theorems. 
Theorem 6.3 (Lin and Takahashi [26]). Let $E$ be a uniformly convex Banach space with a Fréchet differentiable norm and let $C$ be a nonempty subset of $E$. Let $T: C \rightarrow C$ be a generalized nonspreading mapping such that $A(T)=B(T) \neq \emptyset$. Let $R$ be the sunny generalized nonexpansive retraction of $E$ onto $B(T)$. Then for any $x \in C$,

$$
S_{n} x=\frac{1}{n} \sum_{k=0}^{n-1} T^{k} x
$$

converges weakly to an element $q \in A(T)$, where $q=\lim _{n \rightarrow \infty} R T^{n} x$.

Proof. If $\alpha+\beta=-\gamma-\delta=1$ in (3.1), then the mapping is generalizing nonspreading. Therefore we have the desired result from Theorem 6.2.

Theorem 6.4. Let $E$ be a uniformly convex Banach space with a Fréchet differentiable norm. Let $T: E \rightarrow E$ be a generic $(\alpha, \beta, \gamma, \delta, \varepsilon, \zeta)$-generalized nonspreading mapping such that $\alpha+\gamma>0$ and $\varepsilon+\zeta \geq 0$. Assume that $F(T) \neq \emptyset$ and let $R$ be the sunny generalized nonexpansive retraction of $E$ onto $F(T)$. Then for any $x \in E$,

$$
S_{n} x=\frac{1}{n} \sum_{k=0}^{n-1} T^{k} x
$$

converges weakly to an element $q \in F(T)$, where $q=\lim _{n \rightarrow \infty} R T^{n} x$.

Proof. We also know that $\alpha+\gamma>0$ together with $\varepsilon+\zeta \geq 0$ implies that

$$
\phi(T x, u) \leq \phi(x, u)
$$

for all $x \in E$ and $u \in F(T)$. We also have that $A(T)=A(T) \cap E=F(T)$ and $B(T)=B(T) \cap E=F(T)$. Then $A(T)=B(T)$. Therefore we have the desired result from Theorem 6.2.

Theorem 6.5 (Takahashi, Wong and Yao [37]). Let $H$ be a Hilbert space and let $C$ be a nonempty subset of $H$. Let $T: C \rightarrow C$ be a generic generalized hybrid mapping with $A(T) \neq \emptyset$ and let $P$ be the mertic projection of $H$ onto $A(T)$. Then for any $x \in C$,

$$
S_{n} x=\frac{1}{n} \sum_{k=0}^{n-1} T^{k} x
$$

converges weakly to an element $p \in A(T)$.

Proof. As in the proof of Theorem 3.4, a generic generalized hybrid mapping in a Hilbert space is a generic generalized nonspreading mapping in a Banach space. We also note that $A(T)=B(T)$ from the definitions of $A(T)$ and $B(T)$. Since $A(T)$ is a nonempty closed convex subset of $H$, there exists the metric projection of $H$ onto $A(T)$. In a Hilbert space, the metric projection of $H$ onto $A(T)$ is equivalent to the sunny generalized nonexpansive retraction of $E$ onto $A(T)$. Therefore we have the desired result from Theorem 6.2.

Theorem 6.6 (Kocourek, Takahashi and Yao [20]). Let $H$ be a Hilbert space and let $C$ be a nonempty closed convex subset of $H$. Let $T: C \rightarrow C$ be a generalized hybrid mapping with $F(T) \neq \emptyset$ and let $P$ be the mertic projection of $H$ onto $F(T)$. Then for any $x \in C$,

$$
S_{n} x=\frac{1}{n} \sum_{k=0}^{n-1} T^{k} x
$$


converges weakly to an element $p \in F(T)$.

Proof. If $\alpha+\beta=-\gamma-\delta=1$ and $\varepsilon=\zeta=0$ in (3.1), then the mapping is generalizing nonspreading. Then we have from Theorem 6.5 that $\left\{S_{n} x\right\}$ converges weakly to an element $p \in A(T)$. Since $C$ is closed and convex and $\left\{S_{n} x\right\} \subset C$, the weakly convergent point $p$ of $\left\{S_{n} x\right\}$ is in $C$. Hence we have from $A(T) \cap C=F(T)$ that $p \in F(T)$. This completes the proof.

Remark We do not know whether a nonlinear ergodic theorem of Baillon's type for nonspreading mappings holds or not.

\section{WeAK CONVERGENCE THEOREMS}

In this section, we prove a weak convergence theorem of Mann's type for generic generalized nonspreading mappings in a Banach space. Before showing it, we prove the following lemma which is related to [40].

Lemma 7.1. Let $E$ be a smooth and uniformly convex Banach space and let $C$ be a nonempty convex subset of $E$. Let $T: C \rightarrow C$ be a mapping such that $B(T) \neq \emptyset$. Let $\left\{\alpha_{n}\right\}$ be a sequence of real numbers such that $0<\alpha_{n}<1$ and let $\left\{x_{n}\right\}$ be a sequence in $C$ generated by $x_{1}=x \in C$ and

$$
x_{n+1}=\alpha_{n} x_{n}+\left(1-\alpha_{n}\right) T x_{n}, \quad \forall n \in \mathbb{N} .
$$

If $R_{B(T)}$ is a sunny generalized nonexpansive retraction of $C$ onto $B(T)$, then $\left\{R_{B(T)} x_{n}\right\}$ converges strongly to an element $z \in B(T)$.

Proof. Let $m \in B(T)$. Then we have that

$$
\begin{aligned}
\phi\left(x_{n+1}, m\right) & =\phi\left(\alpha_{n} x_{n}+\left(1-\alpha_{n}\right) T x_{n}, m\right) \\
& \leq \alpha_{n} \phi\left(x_{n}, m\right)+\left(1-\alpha_{n}\right) \phi\left(T x_{n}, m\right) \\
& \leq \alpha_{n} \phi\left(x_{n}, m\right)+\left(1-\alpha_{n}\right) \phi\left(x_{n}, m\right) \\
& =\phi\left(x_{n}, m\right) .
\end{aligned}
$$

Then $\lim _{n \rightarrow \infty} \phi\left(x_{n}, m\right)$ exists. Since $\left\{\phi\left(x_{n}, m\right)\right\}$ is bounded, $\left\{x_{n}\right\}$ and $\left\{T x_{n}\right\}$ are bounded. Define $y_{n}=R_{B(T)} x_{n}$ for all $n \in \mathbb{N}$. Since $\phi\left(x_{n+1}, m\right) \leq \phi\left(x_{n}, m\right)$ for all $m \in B(T)$, from $y_{n} \in B(T)$ we have

$$
\phi\left(x_{n+1}, y_{n}\right) \leq \phi\left(x_{n}, y_{n}\right) .
$$

From Lemma 2.6 and (7.1), we have

$$
\begin{aligned}
\phi\left(x_{n+1}, y_{n+1}\right) & =\phi\left(x_{n+1}, R_{B(T)} x_{n+1}\right) \\
& \leq \phi\left(x_{n+1}, y_{n}\right)-\phi\left(R_{B(T)} x_{n+1}, y_{n}\right) \\
& =\phi\left(x_{n+1}, y_{n}\right)-\phi\left(y_{n+1}, y_{n}\right) \\
& \leq \phi\left(x_{n+1}, y_{n}\right) \\
& \leq \phi\left(x_{n}, y_{n}\right) .
\end{aligned}
$$

Thus $\phi\left(x_{n}, y_{n}\right)$ is a convergent sequence. We also have from (7.1) that for all $m \in \mathbb{N}$,

$$
\phi\left(x_{n+m}, y_{n}\right) \leq \phi\left(x_{n}, y_{n}\right) .
$$

From $y_{n+m}=R_{B(T)} x_{n+m}$ and Lemma 2.6, we have

$$
\phi\left(y_{n+m}, y_{n}\right)+\phi\left(x_{n+m}, y_{n+m}\right) \leq \phi\left(x_{n+m}, y_{n}\right) \leq \phi\left(x_{n}, y_{n}\right)
$$


and hence

$$
\phi\left(y_{n+m}, y_{n}\right) \leq \phi\left(x_{n}, y_{n}\right)-\phi\left(x_{n+m}, y_{n+m}\right) .
$$

Using Lemma 2.3, we have that

$$
g\left(\left\|y_{n+m}-y_{n}\right\|\right) \leq \phi\left(y_{n+m}, y_{n}\right) \leq \phi\left(x_{n}, y_{n}\right)-\phi\left(x_{n+m}, y_{n+m}\right),
$$

where $g:[0, \infty) \rightarrow[0, \infty)$ is a continuous, strictly increasing and convex function such that $g(0)=0$. Then the properties of $g$ yield that $R_{B(T)} x_{n}$ converges strongly to an element $z$ of $B(T)$.

Using Lemma 7.1 and the technique developed by [13], we prove the following theorem.

Theorem 7.2. Let $E$ be a uniformly convex and uniformly smooth Banach space and let $C$ be a nonempty convex subset of $E$. Let $T: C \rightarrow C$ be a generic generalized nonspreading mapping such that $A(T)=B(T) \neq \emptyset$. Let $R$ be the sunny generalized nonexpansive retraction of $E$ onto $B(T)$. Let $\left\{\alpha_{n}\right\}$ be a sequence of real numbers such that $0<\alpha_{n}<1$ and $\liminf _{n \rightarrow \infty} \alpha_{n}\left(1-\alpha_{n}\right)>0$. Then a sequence $\left\{x_{n}\right\}$ generated by $x_{1}=x \in C$ and

$$
x_{n+1}=\alpha_{n} x_{n}+\left(1-\alpha_{n}\right) T x_{n}, \quad \forall n \in \mathbb{N}
$$

converges weakly to $z \in A(T)$, where $z=\lim _{n \rightarrow \infty} R x_{n}$.

Proof. Let $m \in B(T)$. Then we have that

$$
\begin{aligned}
\phi\left(x_{n+1}, m\right) & =\phi\left(\alpha_{n} x_{n}+\left(1-\alpha_{n}\right) T x_{n}, m\right) \\
& \leq \alpha_{n} \phi\left(x_{n}, m\right)+\left(1-\alpha_{n}\right) \phi\left(T x_{n}, m\right) \\
& \leq \alpha_{n} \phi\left(x_{n}, m\right)+\left(1-\alpha_{n}\right) \phi\left(x_{n}, m\right) \\
& =\phi\left(x_{n}, m\right) .
\end{aligned}
$$

So $\lim _{n \rightarrow \infty} \phi\left(x_{n}, m\right)$ exists. Then we have that the sequence $\left\{x_{n}\right\}$ is bounded. This implies that $\left\{T x_{n}\right\}$ is bounded. Put $r=\sup _{n \in \mathbb{N}}\left\{\left\|x_{n}\right\|,\left\|T x_{n}\right\|\right\}$. Using Lemma 2.2, we have that

$$
\begin{aligned}
\phi( & \left.x_{n+1}, m\right)=\phi\left(\alpha_{n} x_{n}+\left(1-\alpha_{n}\right) T x_{n}, m\right) \\
\leq & \left\|\alpha_{n} x_{n}+\left(1-\alpha_{n}\right) T x_{n}\right\|^{2}-2\left\langle\alpha_{n} x_{n}+\left(1-\alpha_{n}\right) T x_{n}, J m\right\rangle+\|m\|^{2} \\
\leq & \alpha_{n}\left\|x_{n}\right\|^{2}+\left(1-\alpha_{n}\right)\left\|T x_{n}\right\|^{2}-\alpha_{n}\left(1-\alpha_{n}\right) g\left(\left\|T x_{n}-x_{n}\right\|\right) \\
& -2 \alpha_{n}\left\langle x_{n}, J m\right\rangle-2\left(1-\alpha_{n}\right)\left\langle T x_{n}, J m\right\rangle+\|m\|^{2} \\
= & \alpha_{n}\left(\left\|x_{n}\right\|^{2}-2\left\langle x_{n}, J m\right\rangle+\|m\|^{2}\right) \\
& +\left(1-\alpha_{n}\right)\left(\left\|T x_{n}\right\|^{2}-2\left\langle T x_{n}, J m\right\rangle+\|m\|^{2}\right)-\alpha_{n}\left(1-\alpha_{n}\right) g\left(\left\|T x_{n}-x_{n}\right\|\right) \\
= & \alpha_{n} \phi\left(x_{n}, m\right)+\left(1-\alpha_{n}\right) \phi\left(T x_{n}, m\right)-\alpha_{n}\left(1-\alpha_{n}\right) g\left(\left\|T x_{n}-x_{n}\right\|\right) \\
\leq & \alpha_{n} \phi\left(x_{n}, m\right)+\left(1-\alpha_{n}\right) \phi\left(x_{n}, m\right)-\alpha_{n}\left(1-\alpha_{n}\right) g\left(\left\|T x_{n}-x_{n}\right\|\right) \\
= & \phi\left(x_{n}, m\right)-\alpha_{n}\left(1-\alpha_{n}\right) g\left(\left\|T x_{n}-x_{n}\right\|\right) .
\end{aligned}
$$

Then we obtain

$$
\alpha_{n}\left(1-\alpha_{n}\right) g\left(\left\|T x_{n}-x_{n}\right\|\right) \leq \phi\left(x_{n}, m\right)-\phi\left(x_{n+1}, m\right) .
$$

From the assumption of $\left\{\alpha_{n}\right\}$, we have that

$$
\lim _{n \rightarrow \infty} g\left(\left\|T x_{n}-x_{n}\right\|\right)=0 .
$$


Since $E$ is reflexive and $\left\{x_{n}\right\}$ is bounded, there exists a subsequence $\left\{x_{n_{i}}\right\}$ of $\left\{x_{n}\right\}$ such that $x_{n_{i}} \rightarrow v$ for some $v \in E$. Since $E$ is uniformly convex and uniformly smooth, and $\lim _{n \rightarrow \infty}\left\|T x_{n}-x_{n}\right\|=0$, we have from Proposition 4.1 that $v$ is an attractive point of $T$. Let $\left\{x_{n_{i}}\right\}$ and $\left\{x_{n_{j}}\right\}$ be two subsequences of $\left\{x_{n}\right\}$ such that $x_{n_{i}} \rightarrow u$ and $x_{n_{j}} \rightarrow v$. We know that $u, v \in A(T)$. Put $a=\lim _{n \rightarrow \infty}\left(\phi\left(x_{n}, u\right)-\right.$ $\left.\phi\left(x_{n}, v\right)\right)$. Since

$$
\phi\left(x_{n}, u\right)-\phi\left(x_{n}, v\right)=2\left\langle x_{n}, J v-J u\right\rangle+\|u\|^{2}-\|v\|^{2},
$$

we have $a=2\langle u, J v-J u\rangle+\|u\|^{2}-\|v\|^{2}$ and $a=2\langle v, J v-J u\rangle+\|u\|^{2}-\|v\|^{2}$. From these equalities, we obtain that

$$
\langle u-v, J u-J v\rangle=0 .
$$

Since $E$ is strictly convex, it follows that $u=v$; see [32]. Therefore $\left\{x_{n}\right\}$ converges weakly to an element $u$ of $A(T)$. On the other hand, we know from Lemma 7.1 that $\left\{R_{B(T)} x_{n}\right\}$ converges strongly to an element $z \in B(T)$. From Lemma 2.6, we also have

$$
\left\langle x_{n}-R_{B(T)} x_{n}, J R_{B(T)} x_{n}-J u\right\rangle \geq 0 .
$$

Since $J$ is continuous, we have $\langle u-z, J z-J u\rangle \geq 0$. Since $J$ is monotone, we also have $\langle u-z, J z-J u\rangle \leq 0$. Thus we have $\langle u-z, J z-J u\rangle=0$. Since $E$ is strictly convex, we have $z=u$. This completes the proof.

As in the proofs of Theorems 6.4 and 6.5 , from Theorem 7.2 we can prove the following weak convergence theorems.

Theorem 7.3. Let $E$ be a uniformly convex and uniformly smooth Banach space. Let $T: E \rightarrow E$ be a generic $(\alpha, \beta, \gamma, \delta, \varepsilon, \zeta)$-generalized nonspreading mapping such that $\alpha+\gamma>0$ and $\varepsilon+\zeta \geq 0$. Assume that $F(T) \neq \emptyset$ and let $R$ be the sunny generalized nonexpansive retraction of $E$ onto $F(T)$. Let $\left\{\alpha_{n}\right\}$ be a sequence of real numbers such that $0<\alpha_{n}<1$ and $\liminf _{n \rightarrow \infty} \alpha_{n}\left(1-\alpha_{n}\right)>0$. Then a sequence $\left\{x_{n}\right\}$ generated by $x_{1}=x \in C$ and

$$
x_{n+1}=\alpha_{n} x_{n}+\left(1-\alpha_{n}\right) T x_{n}, \quad \forall n \in \mathbb{N}
$$

converges weakly to $z \in F(T)$, where $z=\lim _{n \rightarrow \infty} R x_{n}$.

Theorem 7.4 (Takahashi, Wong and Yao [37]). Let $H$ be a Hilbert space and let $C$ be a nonempty convex subset of $H$. Let $T: C \rightarrow C$ be a generic generalized hybrid mapping with $A(T) \neq \emptyset$ and let $P$ be the mertic projection of $H$ onto $A(T)$. Let $\left\{\alpha_{n}\right\}$ be a sequence of real numbers such that $0<\alpha_{n}<1$ and $\liminf _{n \rightarrow \infty} \alpha_{n}(1-$ $\left.\alpha_{n}\right)>0$. Then a sequence $\left\{x_{n}\right\}$ generated by $x_{1}=x \in C$ and

$$
x_{n+1}=\alpha_{n} x_{n}+\left(1-\alpha_{n}\right) T x_{n}, \quad \forall n \in \mathbb{N}
$$

converges weakly to $z \in A(T)$, where $z=\lim _{n \rightarrow \infty} P x_{n}$.

Remark We do not know whether a weak convergence theorem of Mann's type for nonspreading mappings holds or not.

Acknowledgements. The first author was partially supported by Grant-in-Aid for Scientific Research No. 23540188 from Japan Society for the Promotion of Science. The second and the third authors were partially supported by the grant NSC 992115-M-110-007-MY3 and the grant NSC 99-2115-M-037-002-MY3, respectively. 


\section{REFERENCES}

[1] Y. I. Alber, Metric and generalized projections in Banach spaces: Properties and applications, in Theory and Applications of Nonlinear Operators of Accretive and Monotone Type (A. G. Kartsatos Ed.), Marcel Dekker, New York, 1996, pp. 15-50.

[2] J.-B. Baillon, Un theoreme de type ergodique pour les contractions non lineaires dans un espace de Hilbert, C.R. Acad. Sci. Paris Ser. A-B 280 (1975), 1511-1514.

[3] E. Blum and W. Oettli, From optimization and variational inequalities to equilibrium problems, Math. Student 63 (1994), 123-145.

[4] F. E. Browder, Convergence theorems for sequences of nonlinear operators in Banach spaces, Math. Z. 100 (1967), 201-225.

[5] P.L. Combettes and A. Hirstoaga, Equilibrium problems in Hilbert spaces, J. Nonlinear Convex Anal. 6 (2005), 117-136.

[6] S. Dhompongsa, W. Fupinwong, W. Takahashi and J.-C. Yao, Fixed Point Theorems for Nonlinear Mappings and Strict Convexity of Banach Spaces, J. Nonlinear Convex Anal. 11 (2010), 175-183.

[7] K. Goebel and W. A. Kirk, Topics in metric fixed point theory, Cambridge University Press, Cambridge, 1990.

[8] N. Hirano, K. Kido and W. Takahashi, Nonexpansive retractions and nonlinear ergodic theorems in Banach spaces, Nonlinear Anal. 12 (1988), 1269-1281.

[9] T. Honda, T. Ibaraki and W. Takahashi, Duality theorems and convergence theorems for nonlineaqr mappings in Banach spaces, Int. J. Math. Statis. 6 (2010), 46-64.

[10] T. Ibaraki and W. Takahashi, Weak and strong convergence theorems for new resolvents of maximal monotone operators in Banach spaces, Adv. Math. Econ. 10 (2007), 51-64.

[11] T. Ibaraki and W. Takahashi, Mosco convergence of sequences of retracts of four nonlinear projections in Banach spaces, in Nonlinear Analysis and Convex Analtsis (W. Takahashi and T. Tanaka Eds.), Yokohama Publishers, Yokohama, 2007, pp. 139-147.

[12] T. Ibaraki and W. Takahashi, A new projection and convergence theorems for the projections in Banach spaces, J. Approx. Theory 149 (2007), 1-14.

[13] T. Ibaraki and W. Takahashi, Weak convergence theorem for new nonexpansive mappings in Banach spaces and its applications, Taiwanese J. Math. 11 (2007), 929-944.

[14] T. Ibaraki and W. Takahashi, Fixed point theorems for new nonlinear mappings of nonexpansive type in Banach spaces, J. Nonlinear Convex Anal. 10 (2009), 21-32.

[15] T. Ibaraki and W. Takahashi, Generalized nonexpansive mappings and a proximal-type algorithm in Banach spaces, Contemp. Math., 513, Amer. Math. Soc., Providence, RI, 2010, pp. $169-180$.

[16] S. Iemoto and W. Takahashi, Approximating fixed points of nonexpansive mappings and nonspreading mappings in a Hilbert space, Nonlinear Anal. 71 (2009), 2082-2089.

[17] S. Itoh and W. Takahashi, The common fixed point theory of singlevalued mappings and multivalued mappings, Pacific J. Math. 79 (1978), 493-508.

[18] S. Kamimura and W. Takahashi, Strong convergence of a proximal-type algorithm in a Banach apace, SIAM J. Optim. 13 (2002), 938-945.

[19] T. Kawasaki and W. Takahashi, Fixed point and nonlinear ergodic theorems for new nonlinear mappings in Hilbert spaces, to appear.

[20] P. Kocourek, W. Takahashi and J. -C. Yao, Fixed point theorems and weak convergence theorems for generalized hybrid mappings in Hilbert spaces, Taiwanese J. Math. 14 (2010), 2497-2511.

[21] P. Kocourek, W. Takahashi and J. -C. Yao, Fixed point theorems and ergodic theorems for nonlinear mappings in Banach spaces, Adv. Math. Econ. 15 (2011), 67-88.

[22] F. Kohsaka and W. Takahashi, Generalized nonexpansive retractions and a proximal-type algorithm in Banach spaces, J. Nonlinear Convex Anal. 8 (2007), 197-209.

[23] F. Kohsaka and W. Takahashi, Existence and approximation of fixed points of firmly nonexpansive-type mappings in Banach spaces, SIAM J. Optim. 19 (2008), 824-835.

[24] F. Kohsaka and W. Takahashi, Fixed point theorems for a class of nonlinear mappings related to maximal monotone operators in Banach spaces, Arch. Math. (Basel) 91 (2008), 166-177.

[25] L.-J. Lin and W. Takahashi, Attractive point theorems and ergodic theorems for nonlinear mappings in Hilbert spaces, Taiwanese J. Math., to appear. 
[26] L.-J. Lin and W. Takahashi, Attractive point theorems for generalized nonspreading mappings in Banach spaces, J. Convex Anal., to appear.

[27] L.-J. Lin, W. Takahashi and Z.-T. Yu, Attractive point theorems and ergodic theorems for 2-generalized nonspreading mappings in Banach spaces, J. Convex Anal., to appear.

[28] W. R. Mann, Mean value methods in iteration, Proc. Amer. Math. Soc. 4 (1953), 506-510.

[29] S. Matsushita and W. Takahashi, A strong convergence theorem for relatively nonexpansive mappings in a Banach space, J. Approx. Theory 134 (2005), 257-266.

[30] S. Reich, A weak convergence theorem for the alternating method with Bregman distances, in Theory and Applications of Nonlinear Operators of Accretive and Monotone Type (A. G. Kartsatos Ed.), Marcel Dekker, New York, 1996, pp. 313-318.

[31] W. Takahashi, A nonlinear ergodic theorem for an amenable semigroup of nonexpansive mappings in a Hilbert space, Proc. Amer. Math. Soc. 81 (1981), 253-256.

[32] W. Takahashi, Nonlinear Functional Analysis, Yokohama Publishers, Yokohama, 2000.

[33] W. Takahashi, Convex Analysis and Approximation of Fixed Points (Japanese), Yokohama Publishers, Yokohama, 2000.

[34] W. Takahashi, Introduction to Nonlinear and Convex Analysis, Yokohama Publishers, Yokohama, 2009.

[35] W. Takahashi, Fixed point theorems for new nonexpansive mappings in a Hilbert space, J. Nonlinear Convex Anal. 11 (2010), 79-88.

[36] W. Takahashi and Y. Takeuchi, Nonlinear ergodic theorem without convexity for generalized hybrid mappings in a Hilbert space, J. Nonlinear Convex Anal. 12 (2011), 399-406.

[37] W. Takahashi, N.-C. Wong and J. C. Yao, Attractive point and weak convergence theorems for new generalized hybrid mappings in Hilbert spaces, to appear.

[38] W. Takahashi and J. C. Yao, Fixed point theorems and ergodic theorems for nonlinear mappings in a Hilbert space, Taiwanese J. Math. 15 (2011), 457-472.

[39] W. Takahashi and J. C. Yao, Nonlinear operators of monotone type and convergence theorems with equilibrium problems in Banach spaces, Taiwanese J. Math. 15 (2011), 787-818.

[40] W. Takahashi and J. C. Yao, Weak and strong convergence theorems for positively homogeneous nonexpansive mappings in Banach spaces, Taiwanese J. Math. 15 (2011), 961-980.

[41] H. K. Xu, Inequalities in Banach spaces with applications, Nonlinear Anal. 16 (1981), 11271138.

(Wataru Takahashi) Department of Mathematical and Computing Sciences, Tokyo InStitute of Technology, Tokyo 152-8552, JaPAN

E-mail address: wataru@is.titech.ac.jp

(Ngai-Ching Wong) Department of Applied Mathematics, National Sun Yat-Sen UniVERSITY, KAOHSIUNG 80424, TAIWAN

E-mail address: wong@math.nsysu.edu.tw

(Jen-Chih Yao) Center for General Education, Kaohsiung Medical University, KaohSIUNG 80702, TAIWAN

E-mail address: yaojc@kmu.edu.tw 